Pacific Journal of Mathematic 


\title{
GENERALIZED ILSTOW AND FEYNMAN INTEGRALS
}

\author{
D. L. Skoug
}

Let $C[a, b]$ denote the space of continuous functions $x(t)$ defined on $[a, b] \ni x(a)=0$. This space is called Wiener space. Using the Wiener integral we define, for each nonnegative integer $M$, what we call the $M$ Ilstow, $M$ complex Wiener, $M$ Feynman, limiting $M$ complex Wiener, and limiting $M$ Feynman integrals of a functional $F(x)$ on $C[a, b]$ and show various relationships which exist between these integrals. In particular we give necessary and sufficient conditions for a finite dimensional functional $F(x)$ to be $M$ Ilstow integrable on $C[a, b]$.

We consider the set of linear functionals $x\left(t_{1}\right), \cdots, x\left(t_{n}\right)$ where $a=t_{0}<t_{1}<\cdots<t_{n}=b$ and obtain conditions on $g_{j}(u) \ni$ the functional

$$
F(x)=g_{1}\left[x\left(t_{1}\right)\right] \cdots g_{n}\left[x\left(t_{n}\right)\right]
$$

is $M$ Ilstow and limiting $M$ Feynman integrable on $C[a, b]$. We then apply these results to the functional

$$
F(t, \xi, x)=\exp \left(\int_{a}^{b} \theta[t-s, x(s)+\xi] d s\right) \sigma[x(t)+\xi]
$$

where $0 \leqq t \leqq t_{0},-\infty<\xi<\infty$ and $x \in C\left[0, t_{0}\right]$ and show that for appropriate functions $\theta(t, \xi)$ and $\sigma(\xi)$, the limiting $M$ Feynman integral $\hat{G}(t, \xi, q)$ of $F(t, \xi, x)$ exists for

$$
(t, \xi, q) \in\left(0, t_{0}\right) \otimes R_{1} \otimes\left\{R_{1}-\{0\}\right\}
$$

and satisfies there the integral equation

$$
\begin{aligned}
\hat{G}(t, \xi, q) & =\left(\frac{-i q}{2 \pi t}\right)^{1 / 2} \int_{-\infty}^{\infty} \sigma(u) \exp \left(\frac{q i(\xi-u)^{2}}{2 t}\right) d u \\
& +\left(\frac{-i q}{2 \pi}\right)^{1 / 2} \int_{0}^{t}(t-s)^{-1 / 2} d s \\
& \left.\times \int_{-\infty}^{\infty} \theta^{\prime} s, u\right) \hat{G}(s, u, q) \exp \left(\frac{q i(\xi-u)^{2}}{2(t-s)}\right) d u
\end{aligned}
$$

For $M=0$ the definitions of the above mentioned integrals reduce to the definitions of the Ilstow, complex Wiener, Feynman, limiting complex Wiener, and limiting Feynman integrals as defined by $R . H$. Cameron in [2]. He used the Ilstow integral as an intermediate integral in his definition of the Feynman integral. The word "Ilstow" is a contraction of "inverse Laplace Stieltjes transform of Wiener's".

Many of the theorems in this paper are generalizations of theorems in [2]. However, the techniques developed in $\S 4$, applied when $M=0$, allow us to reduce the hypothesis of several theorems of [2]. In particular, we obtain a condition for the Ilstow and limiting Feynman 
integrability of $F(x)$ defined by (1.1), that only requires each $g_{i}^{\prime}$ to be essentially of bounded variation on any bounded interval, instead of each $g_{i}^{\prime \prime}$ being continuous as required by Theorem 7.2 of [2].

These more general integrals allow us to weaken the conditions required on $\sigma$ and still obtain the existence of $\hat{G}(t, \xi, q)$.

2. Generalized Ilstow and complex Wiener integrals and the banach space $B(M, \lambda)$.

DeFinition 2.1. Let $M$ be a nonnegative integer. Let $F(\rho x)$ be Lebesque $\otimes$ Wiener integrable on $\left[0, \rho_{0}\right] \otimes C[a, b]$ and assume $\exists f_{M}(s)$ on $[0, \infty) \ni f_{M}(0)=0, f_{M}$ is left continuous and of bounded variation on every bounded interval $\left[0, s_{0}\right], \int_{0}^{\infty} e^{-\lambda s}\left|d f_{M I}(s)\right|<\infty$ for some $\lambda>0$, and for almost all sufficiently large $\lambda$,

$$
\int_{C[a, b]} F\left(\lambda^{-\frac{1}{2}} x\right) d x=\lambda^{M / 2} \int_{0}^{\infty} e^{-\lambda s} d f_{. M}(s) .
$$

Then $F(x)$ will be said to be $M$ Ilstow integrable on $C[a, b]$ and $f_{M}(s)$ is called the $M$ Ilstow integral of $F(x)$ with parameter $s$ and we write $f_{M}(s)=\int_{C[a, b]}^{I L^{M I} W_{s}} F(x) d x$.

Notation. Let $I_{I I}=\{F(x) \mid F(x)$ is $M$ Ilstow integrables on $C[a, b]\}$.

We use the next lemma in the proof of several theorems.

Lemma 2.1. Suppose $f$ is of bounded variation on every bounded interval of $[0, \infty)$ such that for some $\lambda_{0} \geqq 0, \int_{0}^{\infty} e^{-r_{0} s}|d f(s)|<\infty$. Then for $\lambda>\lambda_{0}$

$$
\int_{0}^{\infty} e^{-\lambda s} d f(s)=\lambda^{\frac{1}{2}} \int_{0}^{\infty} e^{-\lambda s} d g(s)
$$

where

$$
g(s)=2 \pi^{-\frac{1}{2}} \int_{0}^{s}(s-\rho)^{1 / 2} d f(\rho) .
$$

In addition, $g$ is absolutely continuous on every bounded interval $\left[0, s_{0}\right]$ and $g(0)=0$. Finally for $\lambda>\lambda_{0}$

$$
\int_{0}^{\infty} e^{-\lambda s}|d g(s)| \leqq \lambda^{-\frac{1}{2}} \int_{0}^{\infty} e^{-\lambda s}|d f(s)| \text {. }
$$

Proof. Same as proof of Lemma 5 on pp. 346-347 of [2]. 
THEOREM 2.1. $I_{M} \subseteq I_{M+1}$ for $M=0,1,2, \cdots$. Furthermore if $F(x) \in I_{M}$ then

$$
f_{M I+1}(s)=2 \pi^{-\frac{1}{2}} \int_{0}^{s}(s-\rho)^{\frac{1}{2}} d f_{M}(\rho)
$$

and

$$
f_{M+2}(s)=2 \pi^{-\frac{1}{2}} \int_{0}^{s}(s-\rho)^{\frac{1}{2}} d f_{M+1}(\rho)=\int_{0}^{s} f_{M}(\rho) d \rho
$$

so that the $(M+2)$ Ilstow integral of $F(x)$ is differentiable a.e. on $[0, \infty)$ and its derivative is $f_{M}(s)$.

Proof. Assume $F(x) \in I_{M}$ with $M$ Ilstow integral $f_{M}(s)$. Define $f_{M+1}(s)$ by equation (2.4) and note that (2.1)-(2.3) imply $F(x) \in I_{M+1}$. To obtain the second equality of (2.5), integrate the middle expression by parts, substitute for $f_{M+1}$ from (2.4) and interchange order of integration.

Definition 2.2. Let $\lambda \neq 0$ be $\ni \operatorname{Re} \lambda \geqq 0$. Let $F(x) \in I_{M}$ with $M$ Ilstow integral $f_{M}(s)$. Then if

$$
\int_{0}^{\infty}\left|e^{-\lambda s}\right|\left|d f_{M}(s)\right|<\infty
$$

we say that $F(x)$ is $M$ complex Wiener integrable on $C[a, b]$ with revar parameter $\lambda(\lambda$ is called the reciprocal variance or revar parameter since in [1], $\lambda^{-1}$ denoted the variance parameter) and we define the value of the $M$ complex Wiener integral to be

$$
\int_{C[a, b]}^{W_{i}^{M}} F(x) d x=\lambda^{M / 2} \int_{0}^{\infty} e^{-\lambda . s} d f_{M}(s)
$$

where if $M$ is odd the square roots have values in the right half plane. If $\operatorname{Re} \lambda=0$ the $M$ complex Wiener integral will be called an $M$ Feynman integral. For $\lambda=-i q, q$ real, we denote the $M$ Feynman integral of $F(x)$ by $\int_{C[a, b]}^{f_{q}^{M}} F(x) d x$.

Note that a functional $F(x)$ is $M$ Feynman integrable if and only if its $M$ Ilstow integral is of bounded variation on $[0, \infty)$. In order to remove this somewhat restrictive condition we shall generalize the above definition slightly.

Definition 2.3. If in Definition 2.2 we replace (2.6) by

$$
\int_{0}^{\infty}\left|e^{-s(\lambda+\eta)}\right|\left|d f_{M}(s)\right|<\infty
$$


for all $\eta \ni \operatorname{Re} \eta>0$ and if the right member of

$$
\int_{C[a, b]}^{\rightarrow W} \underset{\lambda}{W H} F(x) d x=\lambda^{M / 2} \lim _{\substack{\eta \rightarrow 0 \\(\operatorname{Re} \eta>0)}} \int_{0}^{\infty} e^{-s(\lambda+\eta)} d f_{M}(s)
$$

exists, we say that $F(x)$ has a limiting $M$ complex Wiener integral with revar parameter $\lambda$ and we give it the value and notation specified above.

Notation. The symbol $A \rightrightarrows B$ shall mean the existence of $A$ implies the existence of $B$ and the equality $A=B$.

In terms of the analytic Wiener integral, defined by Cameron on p. 289 of [2], and denoted by $\int_{C[a, b]}^{a n W} F(x) d x$, we have the following theorem.

THEOREM 2.2. For $\operatorname{Re} \lambda \geqq 0$

$$
\int_{C[a, b]}^{W_{\lambda}^{M I}} F(x) d x \equiv \int_{C[a, b]}^{\rightarrow W_{\lambda}^{M I}} F(x) d x \equiv \int_{C[a, b]}^{a n W} F(x) d x
$$

and if $F \in I_{I}$ then for almost all sufficiently large positive $\lambda$ we have

$$
\int_{C[a, b]} F\left(\lambda^{-\frac{1}{2}} x\right) d x=\int_{C[a, b]}^{W{ }_{\lambda}^{M}} F(x) d x
$$

Proof. Same as proof of Theorem 1 of [2] as the additional factor $\lambda^{M / 2}$ doesn't affect analyticity.

Notation. Let $W(M, \lambda)=\{F(x) \mid F(x)$ is $M$ complex Wiener integrable on $C[a, b]$ with revar parameter $\lambda\}$. $\vec{W}(M, \lambda)=\{F(x) \mid F(x)$ is limiting $M$ complex Winear integrable on $C[a, b]$ with revar parameter $\lambda\}$.

The following theorem follows directly from Lemma 2.1 and Theorems 2.1 and 2.2.

Theorem 2.3. $W(M, \lambda) \subseteq W(M+1, \lambda)$ for $\operatorname{Re} \lambda>0$ and $M=$ $0,1,2, \cdots . \quad \vec{W}(M, \lambda) \leqq \vec{W}(M+1, \lambda)$ for $\operatorname{Re} \lambda \geqq 0$ and $M=0,1,2, \cdots$. Furthermore if $F(x) \in W(M, \lambda)$ then the $M$ and $(M+1)$ complex Wiener integrals of $F(x)$ are equal (both being equal to the analytic Wiener integral of $F(x)$ by Theorem 2.2). A similar statement holds in case $F(x) \in \vec{W}(M, \lambda)$. 
We now define for $M=0,1,2, \cdots$ and $\lambda \geqq 0$, a space $B(M, \lambda)$ of functionals of $C[a, b]$ and discuss some of its properties. In particular $B(0, \lambda)=B_{\lambda}$ where $B_{\lambda}$ was defined on p. 297 of [2].

Definition 2.4. Let $\lambda \geqq 0$ and $M \in\{0,1,2, \cdots\}$ be given. Let $F(x)$ be a functional defined on $C[a, b] \ni F(\rho x)$ is Lebesque $\otimes$ Wiener measurable in $(\rho, x)$ on $\left[0, \lambda^{-\frac{1}{2}}\right] \otimes C[a, b]$ and $\ni$

$$
N_{\lambda}(F)=\int_{\lambda}^{\infty} e^{\lambda-\rho} d \rho \int_{C[a, b]}\left|F\left(\rho^{-\frac{1}{2}} x\right)\right| d x<\infty
$$

where we interpret $\lambda^{-\frac{1}{2}}$ to be $+\infty$ if $\lambda=0$. Assume further that there exists a left continuous function $f(s)$ of bounded variation on every bounded subinterval of $[0, \infty) \ni$

$$
N_{\lambda}^{\prime}(M, F)=\int_{0}^{\infty} e^{-\lambda s}|d f(s)|<\infty
$$

and such that for almost all $\rho>\lambda$

$$
\rho^{M / 2} \int_{0}^{\infty} e^{-\rho s} d f(s)=\int_{C[a, b]} F\left(\rho^{-\frac{1}{2}} x\right) d x .
$$

Then we say $F \in \widetilde{B}(M, \lambda)$ and define the norm of $F$ to be $N_{\lambda}(M, F)=$ $N_{\lambda}(F)+N_{\lambda}^{\prime}(M, F)$. Note that $N_{\lambda}=N_{\lambda}^{\prime}$ where $N_{\lambda}^{\prime}$ is defined by (2.1) of [2] while $N_{\lambda}^{\prime}(M, \cdot)=N_{\lambda}^{\prime \prime}(\cdot)$ if and only if $M=0$ where $N_{\lambda}^{\prime \prime}$ is defined by (2.2) of [2].

Definition 2.5. We say that two elements $F_{1}$ and $F_{2}$ of $\widetilde{B}(M, \lambda)$ are equivalent if for almost all $(\rho, x)$ on $\left[0, \lambda^{-\frac{1}{2}}\right] \otimes C[a, b]$ we have $F_{1}(\rho x)=F_{2}(\rho x)$. We define $B(M, \lambda)$ as the space of equivalence classes of elements of $\widetilde{B}(M, \lambda)$.

THEOREM 2.4. The space $B(M, \lambda)$ has the following properties:

(a) $B(M, \lambda) \leqq I_{M}$ for $\lambda \geqq 0$ and $M=0,1,2, \cdots$.

(b) $B(M, \lambda)$ is a Banach space with norm $N_{\lambda}(M, \cdot)$.

(c) $B(M, \lambda) \subseteq B(M+1, \lambda)$ for $\lambda>0$, and $M=0,1,2, \cdots$.

(d) $0 \leqq \lambda_{1} \leqq \lambda_{2} \Rightarrow B\left(M, \lambda_{1}\right) \leqq B\left(M, \lambda_{2}\right)$.

Proof. Property (a) follows from the definitions of $B(M, \lambda)$ and $I_{M}$. Property (b) follows from Theorem 2 of [2]. Property (c) follows from equations (2.3), (2.4), (2.9) and (2.10). Property (d) follows as $0 \leqq \lambda_{1} \leqq \lambda_{2}$ implies that $N_{\lambda_{2}}(M, F) \leqq N_{\lambda_{1}}(M, F)$.

3. Finite dimensional functionals. Let $\alpha_{1}(t), \cdots, \alpha_{n}(t)$ be real functions of bounded variation on $[a, b]$ and let $g\left(u_{1}, \cdots, u_{n}\right)$ be a real or complex function defined almost everywhere on $R_{n}$. Let 


$$
F(x)=g\left[\int_{a}^{b} \alpha_{1}(t) d x(t), \cdots, \int_{a}^{b} \alpha_{n}(t) d x(t)\right] .
$$

Then $F(x)$ will be called an " $n$-dimensional functional" on $C[a, b]$. If $g\left(u_{1}, \cdots, u_{n}\right)$ is Lebesque measurable on $R_{n}$ we call $F$ a measurable $n$-dimensional functional. If $\alpha_{1}(t), \cdots, \alpha_{n}(t)$ are an orthonormal set on $[a, b]$, (3.1) will be said to express $F(x)$ in "normalized form".

Our next theorem is a generalization of Theorem 3 of [2] and gives necessary and sufficient conditions that a finite dimensional functional be $M$ Ilstow integrable and a formula for the $M$ Ilstow integral.

Theorem 3. Let $F(x)$ be a measurable n-dimensional functional on $C[a, b]$ expressed in normalized form by (3.1). Let $M \in\{0,1,2, \cdots\}$. Let $K=0$ if $n \leqq M$ while if $n>M$ let $K$ equal $(n-M) / 2$ or $(n+1-M) / 2$ whichever is an integer. Then $F(x)$ is $M$ Ilstow integrable on $C[a, b]$ if and only if the following four conditions are satisfied:

(1) For sufficiently large $\lambda$, the quantity $N_{\lambda}(F)$ is finite, where

$$
\begin{aligned}
N_{\lambda}(F)= & (2 \pi)^{-n / 2} \int_{\lambda}^{\infty} e^{\lambda-\rho} d \rho \int_{R_{n}} \\
& \times\left|g\left(\rho^{-1 / 2} u_{1}, \cdots, \rho^{-1 / 2} u_{n}\right)\right| \exp \left(-\frac{\left(u_{1}^{2}+\cdots+u_{n}^{2}\right)}{2}\right) d u .
\end{aligned}
$$

(2) The function $I_{n}(M, s)$ defined by

$$
I_{n}(M, s)=\int_{u_{1}^{2}+\cdots+u_{M+2 k}^{2}<2 s}^{(M+2 k)} \int g\left(u_{1}, \cdots, u_{n}\right) d u_{1} \cdots d u_{M+2 k}
$$

has $(K-1)$ continuous derivatives all of which of course vanish for $s \leqq 0$.

(3) The $(K-1)$ th derivative $I_{n}^{(K-1)}(M, s)$ has a left hand derivative, which we denote by $I_{n}^{(K)}(M, s)$, which is of bounded variation on every bounded interval and which is a true Kth derivative of $I_{n}(M, s)$ except on the countable set where it has jumps. where

(4) For sufficiently large $\lambda$, the quantity $N_{\lambda}^{\prime}(F, M)$ is finite,

$$
N_{\lambda}^{\prime}(F, M)=\left(\frac{1}{2 \pi}\right)^{(2 k+M) / 2} \int_{0}^{\infty} e^{-\lambda s}\left|d_{s} I_{n}^{(K)}(M, s)\right|
$$

Moreover when conditions (1) through (4) are satisfied we have for $s \geqq 0$

$$
\int_{C[a, b]}^{I L_{W_{s}}^{M}} F(x) d x=\left(\frac{1}{2 \pi}\right)^{(2 k+M) / 2} I_{n}^{(K)}(M, s)
$$


Note that when $K=0$, conditions (2) and (3) reduce to $I_{n}(M, s)$ being of bounded variation on every bounded interval.

Corollary to Theorem 3. For

$$
\begin{aligned}
& M=1,2, \cdots, I_{M-1} \varsubsetneqq I_{M}, B(M-1,0) \\
& \quad \nsubseteq B(M, 0), B(M, 0) \nsubseteq B(M-1,0), W(M-1,0) \\
& \varsubsetneqq W(M, 0) \text { and } W(M, 0) \varsubsetneqq W(M-1,0) .
\end{aligned}
$$

Proof. Let $g\left(u_{1}, \cdots, u_{j}\right)$ be the characteristic function of a $j$ dimensional sphere of radius $\sqrt{2}$ about the origin. Let $\alpha_{1}(t), \cdots$, $\alpha_{j}(t)$ be an orthonormal set on $[a, b]$. Let

$$
F_{j}(x)=g\left[\int_{a}^{b} \alpha_{1}(t) d x(t), \cdots, \int_{a}^{b} \alpha_{j}(t) d x(t)\right] .
$$

Then for $M=1,2, \cdots, F_{M+2}(x) \in I_{M}$ and $F_{M+2}(x) \notin I_{M-1}$. This follows by noting that by use of (2.4) and (3.2) we obtain for $s \geqq 0$

$$
I_{M+2}(M, s)=\frac{(2 \pi)^{(M+2) / 2}}{\Gamma\left(\frac{M+4}{2}\right)} \min \left\{1, s^{(M+2) / 2}\right\}
$$

so that by Theorem $3, F_{M+2}(x) \in I_{M}$ while

$$
\begin{aligned}
I_{M+2}(M-1, s)= & I_{M+2}(M+1, s) \\
& = \begin{cases}\frac{2(2 \pi s)^{(M+3) / 2}}{\Gamma\left(\frac{M+5}{2}\right)} & 0 \leqq s \leqq 1 \\
\frac{2 \sqrt{2}(2 \pi)^{(M+2) / 2}}{\Gamma\left(\frac{M+2}{2}\right)} \int_{0}^{1}(s-\rho)^{1 / 2} \rho^{M / 2} d \rho, & s>1\end{cases}
\end{aligned}
$$

so that $\lim _{s \rightarrow 1+} I_{M+2}^{(2)}(M-1, s)=-\infty$ and hence $F(x) \notin I_{M-1}$.

Similarly for $M=1,2, \cdots$, one can show that

$$
F_{M}(x) \in B(M, 0) \cap W(M, 0) \quad \text { and } \quad F_{M}(x) \notin B(M+1,0) \cup W(M+1,0) .
$$

For $M=3,4, \cdots$ it follows that $F_{M}(x) \in B(M-2,0) \cap W(M-2,0)$ while $F_{M}(x) \notin B(M-3,0) \cup W(M-3,0)$. Finally letting $g\left(u_{1}, u_{2}\right) \equiv 1$ and writing $F(x)$ in form (3.1) one obtains $F(x) \in B(0,0) \cap W(0,0)$ and $F(x) \notin B(1,0) \cup W(1,0)$.

4. Products of functions of $\left(x t_{j}\right)$. In this section we consider the set of linear functionals, $x\left(t_{1}\right), \cdots, x\left(t_{n}\right)$, and obtain conditions under which products of the form 


$$
F(x)=g_{1}\left[x\left(t_{1}\right)\right] \cdots g_{n}\left[x\left(t_{n}\right)\right]
$$

are $M$ Ilstow and limiting $M$ Feynman integrable on $C[a, b] . \quad F(x)$ as given by (4.1) is an $n$-dimensional functional but here we wish to obtain simpler conditions for $M$ Ilstow integrability than those given in Theorem 3. In Theorem 7.1 of [2], R. H. Cameron showed that each $g_{i}^{\prime \prime}$ being continuous and $g_{i}$ and $g_{i}^{\prime \prime}$ both being absolutely integrable on $R$ was sufficient to insure Ilstow integrability. Here we will only require each of the $g_{i}^{\prime}$ s to have a derivative essentially of bounded variation on any bounded interval, and satisfy certain growth conditions.

In Theorem 4 we will find it necessary to express certain LebesqueLebesque Stieltjes iterated integrals as Laplace Stieltjes transforms. We shall now develop the required notation and lemmas to achieve this objective.

LEMMA 4.1. Assume $f(u)$ is absolutely continuous on $[c, d]$ and has a derivative $f^{\prime}(u)$ which is essentially of bounded variation on $[c, d]$. Let $\phi(u) \equiv \operatorname{ess}_{h \rightarrow 0+} \lim f^{\prime}(u+h)$ and assume $\operatorname{Var}(\phi,[c, d])<\infty$. Let $a \in[c, d]$ and let $g(u)=\{f(u)-f(a)\} /(u-a)$ for $u \in[c, d], u \neq a$ and let $g(a)=\phi(a)$. Then $g(u)$ is of bounded variation on [c,d] and $\operatorname{Var}(g,[c, d]) \leqq \operatorname{Var}(\phi,[c, d])$.

Proof. This lemma follows from the observation that for all $u \in[c, d]$ we have $g(u)=\int_{0}^{1} \phi[(u-a) t+a] d t$.

Lemma 4.2. Assume $f_{1}(u), \cdots, f_{n}(u)$ are of bounded variation on $[c, d]$. Let $1 \leqq k \leqq n$ and assume that $g_{1}(u)$ and $g_{2}(u)$ are such that for all $u \in[c, d]$ we have $\left|f_{i}(u)\right| \leqq g_{1}(u)$ for $i=1,2, \cdots, k$, while $\left|f_{i}(u)\right| \leqq g_{2}(u)$ for $i=k+1, \cdots, n$. Let $K_{1}=\max _{u \in[c, d]} g_{1}(u)$ and $K_{2}=\max _{u \in[c, d]} g_{2}(u)$. Then

$$
\begin{aligned}
& \operatorname{Var}\left(\prod_{i=1}^{n} f_{i},[c, d]\right) \leqq K_{1}^{k-1} K_{2}^{n-k-1}[ K_{2} \sum_{1}^{k} \operatorname{Var}\left(f_{i},[c, d]\right)+ \\
&\left.K_{2} \sum_{k+1}^{n} \operatorname{Var}\left(f_{i},[c, d]\right)\right] .
\end{aligned}
$$

Notation. Let $m$ be a positive odd integer. For $i=1,2, \cdots, m$, let $k_{i}$ be a positive constant and let $\phi_{i}(s)=\left(s^{2}\right) /\left(k_{i}\right)$. Let $u_{0} \equiv 0$, $\dot{\psi}_{1}(u)=\sum_{i=1}^{m} \phi_{i}\left(u_{i}-u_{i-1}\right)$, and $\dot{\psi}_{2}(u)=u_{1}^{2}+\dot{\phi}_{2}\left(u_{2}\right)+\sum_{i=3}^{m} \phi_{i}\left(u_{i}-u_{i-1}\right)$. For $\sigma \geqq 0$ and $j=1,2$, let $S_{j}(\sigma)=\left\{u \mid \psi_{j}(u)<\sigma\right\}$. Note that $S_{j}(\sigma)$ is an open $m$-dimensional ellipsoid with its center at the origin.

Lemma 4.3. For $i=1,3, \cdots, m$, assume $g_{i}\left(u_{i}\right)$ is bounded and Lebesque integrable on every bounded interval of $R_{1}$. For $i=2,4, \cdots$, $m-1$, assume $h_{i}\left(u_{i-1}, u_{i}, u_{i+1}\right)$ is Borel measurable in its three vari- 
ables and is right continuous and of bounded variation with respect to $u_{i}$ on every bounded interval $[-2 d, 2 d]$ for any $u_{i-1}, u_{i-1} \in[-d, d]$. For notational purposes we will denote the function $h_{i}\left(u_{i+1}, u_{i}, u_{i+1}\right)$ by $h_{i}\left(u_{i}\right)$. Also assume that $\operatorname{Var}\left(h_{i}(s),-2 d \leqq s \leqq 2 d\right)$ is bounded on $u_{i-1}, u_{i+1} \in[-d, d]$. Then for $j=1,2, A_{j}(\sigma)$ exists and is of bounded variation on $0 \leqq \sigma \leqq \nu$ for any $\nu>0$ where $A_{j}(\sigma)$ is the iterated Lebesque-Lebesque Stieltjes integral

$$
\begin{aligned}
A_{j}(\sigma)= & \int_{-\infty}^{\infty} g_{1}\left(u_{1}\right) d u_{1} \cdots \int_{-\infty}^{\infty} g_{m}\left(u_{m}\right) d u_{m} \int_{-\infty}^{\infty} d_{u_{2}} h_{2}\left(u_{2}\right) \int_{-\infty}^{\infty} d_{u_{4}} h_{4}\left(u_{4}\right) \\
& \cdots \int_{-\infty}^{\infty} \chi_{s_{j}(\sigma)}\left(u_{1}, u_{2}, \cdots, u_{m}\right) d_{u_{m-1}} h_{m-1}\left(u_{m-1}\right)
\end{aligned}
$$

which for notational purposes we shall write as

$$
A_{j}(\sigma)=\int_{0 \leqq \psi_{j}(u)<\sigma}^{(m)} \cdots \int_{\prod_{k=1}}^{(m-1) / 2} g_{2 k-1}\left(u_{2 k-1}\right) \prod_{k=1}^{(m+1) / 2} d_{u_{2 k}} h_{2 k}\left(u_{2 k}\right) d u_{m} \cdots d u_{3} d u_{1} .
$$

Further if $f(\sigma)$ is continuous on $[0, \nu]$ then

$$
\begin{aligned}
\int_{0}^{\nu} f(\sigma) d A_{j}(\sigma)= & \int_{0 \leqq \psi_{j}(u)<\sigma}^{(m)} \cdots \int f\left(\psi_{j}(u)\right)\left(\prod_{k=1}^{(m+1) / 2} g_{2 k-1}\left(u_{2 k-1}\right)\right) \\
& \times\left(\prod_{k=1}^{(m-1) / 2} d_{u_{2 k}} h_{2 k}\left(u_{2 k}\right)\right) d u_{m} d u_{m-2} \cdots d u_{3} d u_{1} .
\end{aligned}
$$

In addition if $f(\sigma)$ is continuous on $[0, \infty)$ then the existence (as a finite number) and absolute convergence of the right side of the following equation implies the existence of the left and equality.

$$
\begin{aligned}
\lim _{C \rightarrow \infty} \int_{0}^{C} f(\sigma) d A_{j}(\sigma)= & \int_{-\infty}^{\infty} \cdots \int_{-\infty}^{\infty} f\left(\psi_{j}(u)\right)\left(\prod_{k=1}^{(m)} g_{2 k-1}\left(u_{2 k-1}\right)\right) \\
& \times\left(\prod_{k=1}^{(m-1) / 2} d_{u_{2 k}} h_{2 k}\left(u_{2 k}\right)\right) d u_{m} \cdots d u_{3} d u_{1} .
\end{aligned}
$$

Proof. As $S_{j}(\sigma)$ is an open set in $R_{m}$ one can show that

$$
\int_{-\infty}^{\infty} d_{u_{2}} h_{2}\left(u_{2}\right) \int_{-\infty}^{\infty} d_{u_{4}} h_{4}\left(u_{4}\right) \cdots \int_{-\infty}^{\infty} \chi_{s^{\prime}(\sigma)}\left(u_{1}, u_{2}, \cdots, u_{m}\right) d_{u_{m-1}} h_{m-1}\left(u_{m-1}\right)
$$

is bounded and Borel measurable in its $(m+1) / 2$ variables $u_{1}, u_{3}, \cdots, u_{m}$. Hence $A_{j}(\sigma)$ exists for $0 \leqq \sigma \leqq \nu$. By breaking up the $g_{i}$ 's and $h_{i}$ 's appropriately one can write $A_{j}(\sigma)$ as the difference of two monotonic increasing functions and so $A_{j}(\sigma)$ is of bounded variation on every bounded interval. Equation (4.2) follows from the general limiting sum type of argument. As $\nu \rightarrow \infty, S_{j}(\sigma) \rightarrow R_{m}$ and so by dominated convergence in each variable we obtain (4.3).

In the following lemma and theorem we will use the notation 
developed by Cameron in Theorem 7.1 of [2].

Lemma 4.4. Assume $h(u)$ is absolutely continuous on every bounded interval of $R_{1}$ and $\phi(u) \equiv \operatorname{ess}_{h \rightarrow 0+} \operatorname{limh}^{\prime}(u+h)$ is of bounded variation on every bounded interval of $R_{1}$. Also assume that for some $A>0, B \geqq 0$ and $0 \leqq \gamma<2$ we have $|h(u)| \leqq A e^{B|u| r}$ and $\int_{-V}^{V}|d \dot{\phi}(s)| \leqq$ $A e^{B V r}$ for all $u \in R_{1}$ and $V>0$. Then for $0 \leqq i<j<k, \lambda>0$ and $u_{i, k}^{j} \in R_{1}$ we have

$$
\begin{aligned}
\int_{-\infty}^{\infty} h\left(u_{j}\right) E_{j}^{i} E_{k}^{j} d u_{j}= & \lambda^{-1 / 2} \Gamma_{i, k}^{j}\left[h\left(u_{j}\right)\right]+\int_{-\infty}^{\infty} E_{j}^{i} E_{k}^{j} \hat{\Gamma}_{i, k}^{j} h\left(u_{j}\right) d u_{j} \\
= & \lambda^{-1 / 2} \Gamma_{i, k}^{j}\left[h\left(u_{j}\right)\right]+\lambda^{-1} \alpha_{i, k}^{j}\left(t_{j}-t_{i}\right) \\
& \times \int_{-\infty}^{\infty} E_{j}^{i} E_{k}^{j} d_{u_{j}}\left[\frac{h\left(u_{j}\right)-h\left(u_{i, k}^{j}\right)}{u_{j}-u_{i, k}^{j}}\right]
\end{aligned}
$$

where the integral in the last term of (4.4) exists both as a Riemann Stieltjes integral and as a Lebesque Stieltjes integral and where $\hat{\Gamma}_{i, k}^{j} h\left(u_{j}\right)=h\left(u_{j}\right)-h\left(u_{i, k}^{j}\right)$.

Proof. The first equality follows directly by noting that

$$
\lambda^{-\frac{1}{2}} E_{k}^{i}=\int_{-\infty}^{\infty} E_{j}^{i} E_{k}^{j} d u_{j}
$$

while the second equality follows from integration by parts which is justified by the above growth conditions.

Theorem 4. Let $a=t_{0}<t_{1}<\cdots<t_{n}=b$. For $i=1,2, \cdots, n-1$ assume $g_{i}(u)$ is absolutely continuous on every bounded subinterval of $R_{1}$ and $\phi_{i}(u)=$ ess $_{h \rightarrow 0+} \lim g_{i}^{\prime}(u+h)$ is of bounded variation on every bounded subinterval of $R_{1}$. Furthermore assume for some $0 \leqq \gamma<2$ and $B>0$

$$
\begin{aligned}
& \left|g_{i}(u)\right| \leqq B \\
& \int_{-\infty}^{\infty}\left|g_{i}(s)\right| d s \leqq B \\
& \int_{-V}^{V}\left|d \phi_{i}(s)\right| \leqq B e^{B V \gamma} \\
& \int_{-V}^{V}\left|d g_{i}(s)\right| \leqq B e^{B V \gamma} \\
& \left|\phi_{i}(u)\right| \leqq B e^{B|u| \gamma}
\end{aligned}
$$

for $i=1,2, \cdots, n-1, u \in R_{1}$ and $V>0$. Furthermore assume

$$
\int_{-\infty}^{\infty}\left|g_{n}(s)\right| d s \leqq B
$$


Let $F(x)$ be given as in (4.1). Then there exists a function $\psi(\lambda)$, analytic for $\operatorname{Re} \lambda>0$, continuous for $\operatorname{Re} \lambda \geqq 0$, and which for real $\lambda>0$ satisfies

$$
\psi(\lambda)=\int_{C[a, b]} F\left(\lambda^{-\frac{1}{2}} x\right) d x .
$$

Furthermore $F(x)$ is $M$ Ilstow and limiting $M$ Feynman integrable for all values of the parameter for $M \geqq 1$ and $F(x) \in B(1, \lambda)$ for all $\lambda>0$.

Proof. By using techniques similar to those used by Cameron in proving Theorem 7.1 of [2] (in particular using (4.4) in place of (7.8) of [2]) we obtain for $\lambda>0$

$$
\begin{aligned}
& \lambda^{-1 / 2} \int_{C[a, b]} F\left(\lambda^{-1 / 2} x\right) d x=\int_{-\infty}^{\infty} \frac{g_{n}\left(u_{n}\right) g_{0, n}^{*}\left(0, u_{n}\right)}{\sqrt{2 \pi(b-a)}} \exp \left(-\frac{\lambda u_{n}^{2}}{2(b-a)}\right) d u_{n} \\
& +\sum_{\substack{\mathfrak{u} \\
j=1}} \int_{q_{j}(n-1)}^{\infty} \int_{-\infty}^{(2 q+1)} \cdots \int_{-\infty}^{\infty} \frac{h^{\sharp}\left(\rho_{1}\right) g_{n}\left(\rho_{q+1}\right)}{\sqrt{2 \pi\left(T_{q+1}^{\prime}-T_{q}\right)}} \\
& \times \prod_{i=1}^{q}\left(\frac{g_{v_{i}}\left(\rho_{i}\right) \alpha_{v_{i}, v_{i+1}}^{v_{i}+1}\left(T_{i}-T_{i}^{\prime}\right)^{1 / 2}}{2 \pi\left(T_{i}^{\prime}-T_{i-1}\right)^{1 / 2}}\right) \\
& \times \exp \left\{-\frac{\lambda}{2}\left(\sum_{i=1}^{q+1} \frac{\left(\rho_{i}-\sigma_{i-1}\right)^{2}}{T_{i}^{\prime}-T_{i-1}}+\sum_{i=1}^{q} \frac{\left(\sigma_{i}-\rho_{i}\right)^{2}}{T_{i}-T_{i}^{\prime}}\right)\right\} \\
& \times\left(\prod_{i=1}^{q} d_{\sigma_{i}} H_{i}^{\sharp}\left(\rho_{i}, \sigma_{i}, \rho_{i+1}\right)\right) d \rho_{q+1} d \rho_{q} \cdots d_{i} \rho_{1} \\
& +\sum_{Q_{3}(n-1)} \int_{-\infty}^{\infty} \stackrel{(2 q+1)}{\cdots} \int_{-\infty}^{\infty} \frac{g_{n}\left(\rho_{q+1}\right) \alpha_{0, v_{2}}^{1}\left(T_{i}-T_{i}^{\prime}\right)}{2 \pi\left[2 \pi\left(T_{q+1}^{\prime}-T_{q}\right)\right]^{1 / 2}} \\
& \times\left(\prod_{i=2}^{q} \frac{g_{v_{i}}\left(\rho_{i}\right)\left(T_{i}-T_{i}^{\prime}\right)^{1 / 2} \alpha_{v_{i}, v_{i+1}}^{v_{i}+1}}{2 \pi\left(T_{i}^{\prime}-T_{i-1}\right)^{1 / 2}}\right) \\
& \times \exp \left\{-\frac{\lambda}{2}\left(\sum_{i=2}^{q+1} \frac{\left(\rho_{i}-\sigma_{i-1}\right)^{2}}{T_{i}^{\prime}-T_{i-1}}+\sum_{i=1}^{q} \frac{\left(\sigma_{i}-\rho_{i}\right)^{2}}{T_{i}-T_{i}^{\prime}}+w^{2}\right)\right\} \\
& \times\left(\prod_{\imath=1}^{q} d_{\sigma_{i}} H_{i}^{\sharp}\left(\rho_{i}, \sigma_{i}, \rho_{i+1}\right)\right) d \rho_{q+1} d \rho_{q} \cdots d \rho_{3} d \rho_{2} d w
\end{aligned}
$$

where $Q_{j}(n-1)$ is the class of all finite sets of integers $\nu_{1}, \nu_{2}, \cdots, \nu_{q+1}$ satisfying

$$
\begin{aligned}
& Q_{1}(n-1)=\left\{\nu_{1}, \cdots, \nu_{q+1} \mid \nu_{1}=1, \nu_{i+1} \geqq \nu_{i}+2, i=1,2, \cdots, q, \nu_{q+1}=n\right\} \\
& Q_{2}(n-1)=\left\{\nu_{1}, \cdots, \nu_{q+1} \mid \nu_{1}>1, \nu_{i+1} \geqq \nu_{i}+2, i=1,2, \cdots, q, \nu_{q+1}=n\right\} \\
& Q_{3}(n-1)=\left\{\nu_{1}, \cdots, \nu_{q+1} \mid \nu_{1}=0, \nu_{i+1} \geqq \nu_{i}+2, i=1,2, \cdots, q, \nu_{q+1}=n\right\} \\
& \text { (4.11) } H_{i}^{\sharp}\left(\rho_{i}, \sigma_{i}, \rho_{i+1}\right) \\
& =\frac{\prod_{k=\nu_{i}+1}^{\nu_{i}+1} g_{k}\left(\alpha_{\nu_{i}+1, \nu_{i+1}}^{k} \sigma_{i}+\beta_{\nu_{i}+1, \nu_{i+1}}^{k} \rho_{i+1}\right)-\prod_{k=\nu_{i+1}}^{\nu_{i+1}-1} g_{k}\left(\alpha_{\nu_{i}, \nu_{i+1}}^{k} \rho_{i}+\beta_{\nu_{i}, \nu_{i+1}}^{k} \rho_{i+1}\right)}{\sigma_{i}-\alpha_{\nu_{i}, \nu_{i+1}}^{\nu} \rho_{i}-\beta_{\nu_{i}, \nu,+1}^{\nu} \rho_{i+1}^{+1}}
\end{aligned}
$$


and where in the sum over $Q_{3}(n-1), \rho_{1} \equiv 0$, and the integrals in the two sums exist both as iterated Riemann-Riemann Stieltjes integrals and as iterated Lebesque-Lebesque Stieltjes integrals.

Now define $\phi_{i}^{\sharp}\left(\sigma_{i}, \rho_{i+1}\right)$ by the equation

$$
\begin{aligned}
\phi_{i}^{\sharp}\left(\sigma_{i}, \rho_{i+1}\right)= & \sum_{k=\nu_{i}^{+1}}^{\nu_{i+1}-1}\left\{\alpha_{\nu_{i}+1, \nu_{i+1}}^{k} \phi_{k}\left(\alpha_{\nu_{i}+1, \nu_{i+1}}^{k} \sigma_{i}+\beta_{\nu_{i}+1, \nu_{i+1}+1}^{k} \rho_{i+1}\right)\right. \\
& \left.\times \prod_{\substack{j=\nu_{i+1} \\
j \neq k}}^{k} g_{j}\left(\alpha_{\nu_{i}+1, \nu_{i+1}}^{j} \sigma_{i}+\beta_{\nu_{i}+1, \nu_{i+1}}^{j} \rho_{i+1}\right)\right\} .
\end{aligned}
$$

Then as $g_{k}^{\prime}=\phi_{k}$ a.e. we see that

$$
\frac{d}{d \sigma_{i}}\left[\prod_{k=\nu_{i+1}}^{\nu_{i+1}-1} g_{k}\left(\alpha_{\nu_{i}+1, \nu_{i+1}}^{k} \sigma_{i}+\beta_{\nu_{i}+1, \nu_{i+1}}^{k} \rho_{i+1}\right)\right]=\phi_{i}^{\sharp}\left(\sigma_{i}, \rho_{i+1}\right) \text { a.e. }
$$

Thus for any $d>0$ and $\rho_{i}, \rho_{i+1} \in[-d, d]$ we apply Lemma 4.1 and 4.2 and obtain

$$
\begin{aligned}
\operatorname{Var} & \left(H_{i}^{\sharp}\left(\rho_{i}, s, \rho_{i+1}\right),-2 d \leqq s \leqq 2 d\right) \\
& \leqq \operatorname{Var}\left(\dot{\phi}_{i}^{\sharp}\left(s, \rho_{i+1}\right),-2 d \leqq s \leqq 2 d\right) \\
& \leqq\left(\nu_{i+1}-\nu_{i}-1\right)^{2} B^{\nu_{i+1} \nu_{i-1}^{-1}} e^{2 B}(3 d)^{\gamma} .
\end{aligned}
$$

Hence we see that $H_{i}^{*}$ is of bounded variation as a function of $\sigma_{i}$ on every bounded interval and as $0 \leqq \gamma<2$ the integrals on the right side of (4.10) converge absolutely for $\lambda>0$.

Next we apply Lemma 4.3 to the right side of (4.10) and obtain by use of (4.3)

$$
\int_{C[a, b]} F\left(\lambda^{-\frac{1}{2}} x\right) d x=\lambda^{\frac{1}{2}} \int_{0}^{\infty} e^{-\lambda u} d f(u)
$$

where $f(u)=f_{1}(u)+f_{2}(u)+f_{3}(u)$ and

$$
\begin{aligned}
& f_{1}(u)=\int_{V_{1}(u)} \frac{g_{n}(s) g_{0, n}^{\sharp \sharp}(0, s) d s}{\sqrt{2 \pi(b-a)}} \\
& f_{2}(u)=\sum_{\substack{\bigcup_{j=1}^{2} \\
Q_{j}(n-1)}} \int_{V_{2}(u)} \stackrel{(2 q+1)}{\cdots} \int \frac{g_{n}\left(\rho_{q+1}\right) h^{\sharp}\left(\rho_{1}\right)}{\left[2 \pi\left(T_{q+1}^{\prime}-T_{q}\right)\right]^{1 / 2}}\left(\prod_{i=1}^{q} \frac{g_{\nu_{i}}\left(\rho_{i}\right) \alpha_{\nu_{i}, \nu_{i+1}}^{\nu_{i}+1}\left(T_{i}-T_{i}^{\prime}\right)^{1 / 2}}{2 \pi\left(T_{i}^{\prime}-T_{i-1}\right)^{1 / 2}}\right) \\
& \times\left(\prod_{i=1}^{q} d_{\sigma_{i}} H_{i}^{\sharp}\left(\rho_{i}, \sigma_{i}, \rho_{i+1}\right)\right) d \rho_{q+1} \cdots d \rho_{2} d \rho_{1} \\
& f_{3}(u)=\sum_{Q_{3}(n-1)} \int_{V_{3}(u)} \stackrel{(2 q+1)}{\cdots} \int \frac{g_{n}\left(\rho_{q+1}\right) \alpha_{0, \nu_{2}}^{1}\left(T_{1}-T_{1}^{\prime}\right)^{1 / 2}}{2 \pi\left[2 \pi\left(T_{q+1}^{\prime}-T_{q}\right)\right]^{1 / 2}} \\
& \times\left(\prod_{i=2}^{q} \frac{g_{\nu_{i}}\left(\rho_{i}\right) \alpha_{\nu_{i} \nu_{i+1}+1}^{\nu_{i}+1}\left(T_{i}-T_{i}^{\prime}\right)^{1 / 2}}{2 \pi\left(T_{i}^{\prime}-T_{i-1}\right)^{1 / 2}}\right) \\
& \times\left(\prod_{i=1}^{q} d_{\sigma_{i}} H_{i}^{\sharp}\left(\rho_{i}, \sigma_{i}, \rho_{i+1}\right)\right) d \rho_{q+1} \cdots d \rho_{2} d w
\end{aligned}
$$




$$
\begin{aligned}
V_{1}(u)= & \left\{s \mid s^{2}<2 u(b-a)\right\} \\
V_{2}(u)= & \left\{\left[\rho_{1}, \cdots, \rho_{q+1}, \sigma_{1}, \cdots, \sigma_{q}\right] \mid\right. \\
& \left.\times \sum_{i=1}^{q+1} \frac{\left(\rho_{i}-\sigma_{i-1}\right)^{2}}{T_{i}^{\prime}-T_{i-1}}+\sum_{i=1}^{q} \frac{\left(\sigma_{i}-\rho_{i}\right)^{2}}{T_{i}-T_{i}^{\prime}}<2 u\right\} \\
V_{3}(u)= & \left\{\left[w, \rho_{2}, \cdots, \rho_{q+1}, \sigma_{1}, \cdots, \sigma_{q}\right] \mid w^{2}\right. \\
& \left.+\sum_{i=2}^{q+1} \frac{\left(\rho_{i}-\sigma_{i-1}\right)^{2}}{T_{i}^{\prime}-T_{i-1}}+\sum_{i=1}^{q} \frac{\left(\sigma_{i}-\rho_{i}\right)^{2}}{T_{i}-T_{i}^{\prime}}<2 u\right\} .
\end{aligned}
$$

In order to show that $F(x)$ is $M$ Ilstow integrable for $M=1$ we need to obtain bounds on the variation of $f(u)$ for $u \geqq 0$. First we note that for $j=1,2$, and $3, f_{j}(u)$ is left continuous (by definition of $V_{j}(u)$ ) and of bounded variation on every bounded subinterval of $[0, \infty)$. Now for $j=1,2$ and 3 , let $\hat{f}_{j}(u)$ denote what we obtain be replacing $g_{0, n}^{\text {辛}}, h^{\sharp}, g_{\nu_{i}}$, and $d_{\sigma_{i}} H_{i}^{\sharp}\left(\rho_{i}, \sigma_{i}, \rho_{i+1}\right)$ by $\left.\mid g_{0, n}^{\sharp \sharp}\right\},\left|h^{\sharp}\right|,\left|g_{\nu_{i}}\right|$ and $\left|d_{\sigma_{i}} H_{i}^{\sharp}\left(\rho_{i}, \sigma_{i}, \rho_{i+1}\right)\right|$ respectively in $f_{j}(u)$. Then clearly $\hat{f}_{j}(u) \uparrow$ and for $0 \leqq u_{1}<u_{2}<\infty$ we have

$$
\operatorname{Var}\left(f_{j},\left[u_{1}, u_{2}\right]\right) \leqq \operatorname{Var}\left(\hat{f}_{j},\left[u_{1}, u_{2}\right]\right)=\hat{f}_{j}\left(u_{2}\right)-\hat{f}_{j}\left(u_{1}\right)
$$

Thus we see that it will suffice to obtain bounds on $\hat{f}_{j}(u)$. First note that for all $u \geqq 0$

$$
\hat{f}_{1}(u) \leqq \int_{-\infty}^{\infty} \frac{\left|g_{n}(s) g_{0, n}^{\sharp}(0, s)\right|}{\sqrt{2 \pi(b-a)}} d s \leqq B^{n}[2 \pi(b-a)]^{-1 / 2} .
$$

Next we see that $V_{2}(u) \subseteq \hat{V}_{2}(u)$ and $V_{3}(u) \subseteq \hat{V}_{3}(u)$ where

$$
\begin{aligned}
& \hat{V}_{2}(u)=\left\{\left[\rho_{1}, \cdots, \rho_{q+1}, \sigma_{1}, \cdots, \sigma_{q}\right]|| \rho_{i}|\leqq \hat{u},| \sigma_{i} \mid \leqq 2 \widehat{u}\right\} \\
& \hat{V}_{3}(u)=\left\{\left[w, \rho_{2}, \cdots, \rho_{q+1}, \sigma_{1}, \cdots, \sigma_{q}\right]|| \rho_{i}|\leqq \hat{u},| \sigma_{i} \mid \leqq 2 \widehat{u}, w^{2} \leqq 2 u\right\}
\end{aligned}
$$

and $\widehat{u}=[2 u(b-a)]^{\frac{1}{2}}$. Thus for $\left[\rho_{1}, \cdots, \rho_{q+1}, \sigma_{1}, \cdots, \sigma_{q}\right] \in \hat{V}_{2}(u)$ we have $\alpha_{\nu_{\nu}, \nu i+1}^{\nu i+1} \rho_{i}+\beta_{\nu_{i}, \nu i+1}^{\nu i+1} \rho_{i+1}^{\nu} \in[-2 \hat{u}, 2 \hat{u}]$ for $i=1,2, \cdots, q$, so that by Lemmas 4.1 and 4.2 and equation (4.13) we see that

$$
\begin{aligned}
\operatorname{Var}\left(H_{i}^{\sharp}(\right. & \left.\left.\rho_{i}, s, \rho_{i+1}\right),-2 \hat{u} \leqq s \leqq 2 \widehat{u}\right) \\
& \leqq\left(\nu_{i+1}-\nu_{i}-1\right)^{2} B^{\nu_{i+1} \nu^{-}{ }^{-1}} \exp 2 B(3 \hat{u})^{r} .
\end{aligned}
$$

Hence by replacing $V_{2}(u)$ by $\hat{V}_{2}(u)$ in $\hat{f}_{2}(u)$ we obtain 


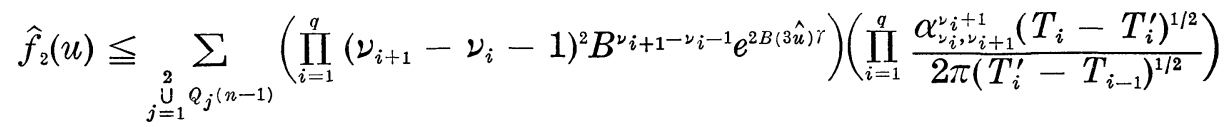

$$
\begin{aligned}
& \times \int_{-\hat{u}}^{\hat{u}} \stackrel{(q+1)}{\hat{u}} \frac{\left|h^{\sharp}\left(\rho_{1}\right) g_{n}\left(\rho_{q+1}\right)\right|}{\left[2 \pi\left(T_{q+1}^{\prime}-T_{q}\right)\right]^{1 / 2}}\left(\prod_{i=1}^{q}\left|g_{\nu_{i}}\left(\rho_{i}\right)\right|\right) d \rho_{q+1} \cdots d \rho_{1} \\
& \leqq \sum_{\substack{j=1 \\
j=1}} \frac{B^{n} e^{2 B q(n-1)}}{\left[2 \pi\left(T_{q+1}^{\prime}-T_{q}\right)\right]^{1 / 2}} \prod_{i=1}^{q} \frac{\left(T_{i}-T_{i}^{\prime}\right)^{1 / 2}\left(\nu_{i+1}-\nu_{i}-1\right)^{2} \alpha_{\nu_{i} \nu_{i+1}+1}}{2 \pi\left(T_{i}^{\prime}-T_{i-1}\right)^{1 / 2}} \\
& <\frac{2^{n-1} B^{n} e^{B n\left[3(2 u(b-a))^{1 / 2}\right] \gamma}}{3\left[2 \pi\left(t_{1}-a\right)\right]^{1 / 2}}
\end{aligned}
$$

since $q \leqq(n / 2)$, while by (2.6) of [4] and (7.38) of [2],

$$
\begin{aligned}
& \sum_{\substack{i=1 \\
j=1}}\left[2 \pi\left(T_{q+1}^{\prime}-T_{q}\right)\right]^{-1 / 2} \prod_{i=1}^{q} \frac{\left(T_{i}-T_{i}^{\prime}\right)^{1 / 2}\left(\nu_{i+1}-\nu_{i}-1\right)^{2} \alpha_{\nu_{i}, \nu i+1}^{\nu+1}}{2 \pi\left(T_{i}^{\prime}-T_{i-1}\right)^{1 / 2}} \\
& \leqq\left[2 \pi\left(t_{1}-a\right)\right]^{-1 / 2} \sum_{j=1}^{\underset{j}{U} Q_{g_{j}}(n-1)}\left(\frac{1}{2 \pi}\right)^{q} \prod_{i=1}^{q}\left(\nu_{i+1}-\nu_{i}-1\right)^{2} \\
& \leqq\left[2 \pi\left(t_{1}-a\right)\right]^{-1 / 2} 3^{-1} \exp \left\{(n-1) \pi^{-1 / 3}\right\} \\
& <2^{n-1} 3^{-1}\left[2 \pi\left(t_{1}-a\right)\right]^{-1 / 2} \text {. }
\end{aligned}
$$

Thus $0 \leqq \gamma<2$ implies $\int_{0}^{\infty} e^{-\lambda u} d \hat{f}_{2}(u)<\infty$ for $\lambda>0$.

Similarly we can show that for $u>0$

$$
\widehat{f}_{3}(u) \leqq 2^{(2 n+-1) / 2} B^{n} \exp \left(n B\left(3[2 u(b-a)]^{\frac{1}{2}}\right)^{\gamma}\right) 3^{-1} \pi^{-\frac{1}{2}}[2 u(b-a)]^{\frac{1}{2}}
$$

and hence for $\lambda>0, \int_{0}^{\infty} e^{-\lambda u} d \hat{f}_{3}(u)<\infty$. Thus for $\lambda>0$

$$
N_{\lambda}(F)=\int_{\lambda}^{\infty} e^{\lambda-\rho} d \rho \int_{C[a, b]}\left|F\left(\rho^{-\frac{1}{2}} x\right)\right| d x \leqq B^{n}
$$

and

$$
N_{\lambda}^{\prime}(1, F)=\int_{0}^{\infty} e^{-\lambda u}|d f(u)| \leqq \sum_{j=1}^{3} \int_{0}^{\infty} e^{-i u} d \hat{f}_{j}(u)<\infty
$$

so that $F(x) \in B(1, \lambda)$ for $\lambda>0$ and in particular, $F(x)$ is $M$ Ilstow integrable for $M \geqq 1$.

Next for $\lambda \ni \operatorname{Re} \lambda \geqq 0$ let

$$
\psi(\lambda)=\lambda^{n / 2} \int_{-\infty}^{\infty} \stackrel{(n)}{\cdots} \int_{-\infty}^{\infty} g_{1}\left(u_{1}\right) \cdots g_{n}\left(u_{n}\right) E_{1}^{0} \cdots E_{n}^{n-1} d u_{n} \cdots d u_{1} .
$$

Then by (7.5) of [2] we have $\psi(\lambda)=\int_{C[a, b]} F\left(\lambda^{-\frac{1}{2}} x\right) d x$ for all $\lambda>0$. Clearly $\psi(\lambda)$ is analytic for $\operatorname{Re} \lambda>0$ and continuous for $\operatorname{Re} \lambda \geqq 0$. 
Hence $\int_{C[a, b]} F\left(\lambda^{-\frac{1}{2}} x\right) d x$ has an analytic extension throughout the right half plane continuous up to the imaginary axis. But for almost all $\rho>0, \int_{C[a, b]} F\left(\rho^{-\frac{1}{2}} x\right) d x=\rho^{\frac{1}{2}} \int_{0}^{\infty} e^{-\rho s} d f(s)$. Thus as $\int_{0}^{\infty} e^{-\rho s}|d f(s)|<\infty$ for all $\rho>0$ we know that $\rho^{\frac{1}{2}} \int_{0}^{\infty} e^{-\rho s} d f(s)$ is analytic in the right half plane and equal to the analytic extension of $\int_{C[a, b]} F\left(\rho^{-\frac{1}{2}} x\right) d x$ there. Thus $\lambda^{\frac{1}{2}} \int_{0}^{\infty} e^{-\lambda s} d f(s)$ approaches a limiting value on the imaginary axis when we approach from the right hand plane and so the limiting $M$ Feynman integral of $F$ exists for $M \geqq 1$ which concludes proof of theorem.

If we restrict the bounds on $\operatorname{Var} \dot{\phi}_{i}$, $\operatorname{Var} g_{i}$, and $\phi_{i}$ we obtain the following corollary which proves useful in a later section.

Corollary to Theorem 4. Assume the hypotheses of Theorem 4 are satisfied where conditions (4.7)-(4.9) are replaced by

$$
\begin{aligned}
& \int_{-V}^{V}\left|d \dot{\phi}_{i}(s)\right| \leqq B\left[1+V^{d}\right], \\
& \int_{-V}^{V}\left|d g_{i}(s)\right| \leqq B\left[1+V^{d}\right], \quad \text { and } \quad\left|\dot{\phi}_{i}(u)\right| \leqq B\left[1+|u|^{d}\right]
\end{aligned}
$$

for $i=1,2, \cdots, n-1, u \in R_{1}, V>0$, and $d>0$. Then the conclusions of Theorem 4 hold and in addition we have $N_{\lambda}(F) \leqq B^{n}$ and

$$
\begin{aligned}
N_{\lambda}^{\prime}(1, F) \leqq & B^{n}[2 \pi(b-a)]^{\frac{1}{2}} \\
& +\frac{2^{n-1} B^{n}\left(1+3^{d}\right)^{n}}{3\left[2 \pi\left(t_{1}-a\right)\right]^{1 / 2}}\left(1+\left[\frac{2(b-a)}{\lambda}\right]^{d n / 2} \Gamma\left[\frac{d n+2}{2}\right]\right) \\
& +\frac{2^{n+1} B^{n}\left(1+3^{d}\right)^{n}}{3(2 \pi)^{1 / 2}}\left(1+\left[\frac{2(b-a)}{\lambda}\right]^{(d n+1) / 2} \Gamma\left[\frac{d n+3}{2}\right]\right) .
\end{aligned}
$$

REMARK. Let us point out at this time that the techniques used in Theorem 4 allow us to strengthen Theorems 7.1 and 7.2 of [2]. For, clearly, if we put the same restrictions on $g_{n}$ as we put on each $g_{i}, i=1,2, \cdots, n-1$, then the conclusions of Theorem 4 hold for $M=0$. Also if condition (4.6) was relaxed so that

$$
\int_{-V}^{V}\left|g_{i}(s)\right| d s \leqq B e^{B V^{r}}, \quad \text { for } i=1,2, \cdots, n \text {, }
$$

then one can show, using the techniques of Theorem 4 , that $F \in I_{0}$ and $F \in B(0, \lambda)$ for all $\lambda>0$. However these same techniques do not imply $F \in \vec{W}(M, 0)$ since in this case $\psi(\lambda)$ as defined by (4.15) is not necessarily continuous up to $\operatorname{Re} \lambda=0$.

5. Entire functions of integrals of $\theta(s, x(s))$. Our first theorem 
deals with $M$ Ilstow and limiting $M$ Feynman integrability of functionals of the form

$$
F^{\sharp}(x)=\Phi\left[\int_{a}^{b} \theta(s, x(s)) d s\right] g(x(b)) .
$$

THEOREM 5.1. Assume $\Phi(z)$ is an entire function satisfying $|\Phi(z)| \leqq A e^{B|z|}$. For each $s \in[a, b]$ assume $\theta(s, u)$ is absolutely continuous as a function of $u$ on every bounded interval of $R_{1}$. Also, for each $s \in[a, b]$, assume $\hat{\theta}(s, u)=\operatorname{ess}_{h \rightarrow 0+} \lim \theta_{u}(s, u+h)$ is of bounded variation as a function of $u$ on every bounded interval of $R_{1}$. Furthermore assume $|\theta(s, V)|, \int_{-\infty}^{\infty}|g(w)| d w$, and $\int_{-\infty}^{\infty}|\theta(s, w)| d w$ are bounded by $B$ while $|\hat{\theta}(s, V)|$,

$$
\int_{-V}^{V}\left|d_{w} \theta(s, w)\right| \text { and } \int_{-V}^{V}\left|d_{w} \hat{\theta}(s, w)\right|
$$

are bounded by $B\left(1+|V|^{d}\right)$ for each $s \in[a, b], V \in R_{1}$ and some $d \ni 0 \leqq d<2$. Then for $M=1,2, \cdots$, the functional $F^{\sharp}(x)$ as defined by (5.1) is $M$ Ilstow and limiting $M$ Feynman integrable for all values of the parameter and $F^{\sharp}(x) \in B(M, \lambda)$ for all $\lambda>0$.

Proof. Since $\Phi(z)$ is an entire function such that $|\Phi(z)| \leqq A e^{B|z|}$ we obtain, by use of Cauchy's inequality, that $\Phi(z)=\sum_{n=0}^{\infty} a_{n} z^{n}$ for all $z$ where $a_{0}=1$ and for $n>0$

$$
\left|a_{n}\right| \leqq A\left(\frac{B e}{n}\right)^{n}
$$

Thus on $C[a, b]$ we have $F^{\sharp}(x)=\sum_{n=0}^{\infty} a_{n} F_{n}^{\sharp}(x)$ where

$$
F_{n}^{\sharp}(x)=g(x(b))\left[\int_{a}^{b} \theta(s, x(s)) d s\right]^{n}
$$

for $n=0,1,2, \cdots$. Now for $n>0$ we have

$$
F_{n}^{\sharp}(x)=\int_{a}^{b} \stackrel{(n)}{\cdots} \int_{a}^{b} F_{n}(x ; s) d s_{1} \cdots d s_{n}
$$

where

$$
F_{n}(x ; s)=F_{n}\left(x ; s_{1}, \cdots, s_{n}\right)=g(x(b)) \prod_{i=1}^{n} \theta\left(s_{i}, x\left(s_{i}\right)\right)
$$

when $s_{1}, \cdots, s_{n}$ are distinct elements of $(a, b)$ but $F_{n}(x ; s)=0$ when $s_{1}, \cdots, s_{n}$ are elements of $[a, b]$ but $a, s_{1}, \cdots, s_{n} b$ are not distinct.

We note that for each $n>0$ and each $s \in[a, b]^{n}, F_{n}(x ; s)$ satisfies the hypotheses of the Corollary to Theorem 4 . Hence for each $s$ in $[a, b]^{n}$ we have for $\lambda>0$ 


$$
\begin{aligned}
& N_{\lambda}\left(F_{n}(\cdot ; s)\right) \leqq B^{n+1}, \\
& N_{\lambda}^{\prime}\left(1, F_{n}(\cdot ; s)\right) \leqq H(s) \\
& \quad \equiv B^{n+1}\left[2 \pi(b-a]^{-\frac{1}{2}}+K_{1}(n)+K_{2}(n)\left[\min \left\{s_{1}, \cdots, s_{n}\right\}-a\right]^{-\frac{1}{2}}\right.
\end{aligned}
$$

where

$$
\begin{aligned}
K_{1}(n)= & 2^{n+2} B^{n+1}\left(1+3^{d}\right)^{n+1} 3^{-1}(2 \pi)^{-1 / 2} \\
& \times\left(1+\left[\frac{2(b-a)}{\lambda}\right]^{[d(n+1)+1] / 2} \Gamma\left[\frac{d(n+1)+3}{2}\right]\right)
\end{aligned}
$$

and

$$
\begin{aligned}
K_{2}(n)= & 2^{n} B^{n+1}\left(1+3^{d}\right)^{n+1} 3^{-1}(2 \pi)^{-1 / 2} \\
& \times\left(1+\left[\frac{2(b-a)}{\lambda}\right]^{[d(n+1)] / 2} \Gamma\left[\frac{d(n+1)+2}{2}\right]\right) .
\end{aligned}
$$

Moreover for each $s, F_{n}(x ; s)$ is $M$ Ilstow and limiting $M$ Feynman integrable for each value of the parameter for $M=1,2, \cdots$, and $F_{n}(x ; s) \in B(1, \lambda)$ for all $\lambda>0$. By Corollary to Theorem 6.4 of [2] (which can be generalized to apply here) we obtain

$$
\begin{aligned}
N_{\lambda}^{\prime}\left(1, F_{n}^{\sharp}\right) & \leqq \int_{a}^{b} \cdots \int_{a}^{b} H(s) d s_{1} \cdots d s_{n} \\
& <B^{n+1}(b-a)^{n}[2 \pi(b-a)]^{-\frac{1}{2}}+(b-a)^{n} K_{1}(n)+2 n(b-a)^{n} K_{2}(n)
\end{aligned}
$$

where the last inequality follows from (8.12) of [2]. Clearly $N_{\lambda}\left(F_{n}^{*}\right) \leqq B^{n+1}(b-a)^{n}$ so that for $\lambda>0$

$$
\begin{aligned}
N_{\lambda}\left(1, \sum_{n=0}^{\infty} a_{n} F_{n}^{\sharp}\right) \leqq & \sum_{n=0}^{\infty} N_{\lambda}\left(1, a_{n} F_{n}^{\sharp}\right) \\
\leqq & \left([2 \pi(b-a)]^{-\frac{1}{2}}+1\right) \sum_{n=0}^{\infty} B^{n+1}(b-a)^{n} a_{n} \\
& +\sum_{n=0}^{\infty}(b-a)^{n} a_{n}\left[K_{1}(n)+2 n K_{2}(n)\right]
\end{aligned}
$$

where $K_{1}(0)=K_{2}(0)=1$. By use of (5.2) the above series converge for $0 \leqq d<2$ by the ratio test and thus the series $\sum_{n=0}^{\infty} a_{n} F_{n}^{\sharp}$ converges in the $B(1, \lambda)$ topology for each $\lambda>0$ and since the series also converges uniformly to $F^{\sharp}$ it converges in the $B(1, \lambda)$ topology to $F^{*}$ and so $F^{*} \in B(1, \lambda)$ for all $\lambda>0$. In particular $F^{*}$ is $M$ Ilstow integrable for $M=1,2, \cdots$.

Next we will show that $F^{*}$ is limiting $M$ Feynman integrable for $M=1$. First apply Corollary to Theorem 4 to the functionals $F_{n}(x ; s)$ and obtain functions $\psi_{n}(\lambda, s)$ which are analytic for $\operatorname{Re} \lambda>0$ and continuous for $\operatorname{Re} \lambda \geqq 0$ for each $n>0$ and $s \in[a, b]^{n}$. In addition for $\operatorname{Re} \lambda \geqq 0$ we have 


$$
\psi_{n}(\lambda, s)=\lambda^{(n+1) / 2} \int_{-\infty}^{\infty} \stackrel{(n+1)}{\cdots} \int_{-\infty}^{\infty} g\left(u_{n+1}\right) \prod_{i=1}^{n} \theta\left(s_{i}, u_{i}\right) E_{1}^{0} E_{2}^{1} \cdots E_{n}^{n+1} d u_{n+1} \cdots d u_{1}
$$

so that for $s \in[a, b]^{n}$ and $\operatorname{Re} \lambda \geqq 0,\left|\psi_{n}(\lambda, s)\right| \leqq|\lambda|^{(n+1) / 2_{3} B^{n+1}}$. Hence letting

$$
\psi_{n}^{\sharp}(\lambda)=\int_{a}^{b} \stackrel{(n)}{\cdots} \int_{a}^{b} \psi_{n}(\lambda, s) d s_{1} \cdots d s_{n}
$$

we have

$$
\left|\psi_{n}^{\sharp}(\lambda)\right| \leqq|\lambda|^{(n+1) / 2_{B} n+1}(b-a)^{n}
$$

for $\operatorname{Re} \lambda \geqq 0$ and $n=1,2, \cdots$. Clearly $\psi_{n}^{*}(\lambda)$ is analytic for $\operatorname{Re} \lambda>0$ and continuous for $\operatorname{Re} \lambda \geqq 0$ for $n=1,2, \cdots$. Similarly we apply Corollary to Theorem 4 to $F_{0}^{*}$ and obtain $\psi_{0}^{*}(\lambda)$ with the same properties.

Next we see that as the series $\sum_{n=0}^{\infty} a_{n} F_{n}^{*}(x)$ converges uniformly in $x$ over $C[a, b]$ we have for each real $\lambda>0$

$$
\begin{aligned}
\int_{C[a, b]} F^{\sharp}\left(\lambda^{-\frac{1}{2}} x\right) d x & =\sum_{n=0}^{\infty} a_{n} \int_{C[a, b]} F_{n}^{\sharp}\left(\lambda^{-\frac{1}{2}} x\right) d x \\
& =\sum_{n=0}^{\infty} a_{n} \psi_{n}^{\sharp}(x) .
\end{aligned}
$$

But by (5.2) and (5.3) the last member of (5.4) converges uniformly in $\lambda$ for any closed subset of $\operatorname{Re} \lambda \geqq 0$ and hence the first member of (5.4) has an analytic extension throughout $\operatorname{Re} \lambda>0$. New let $f_{1}^{\#}$ denote the $M$ Ilstow integral of $F^{*}$ for $M=1$. Then

$$
N_{\grave{\lambda}}^{\prime}\left(1, F^{\sharp}\right)=\int_{0}^{\infty} e^{-\lambda s}\left|d f_{1}^{\sharp}(s)\right|
$$

for each $\lambda>0$ and for almost all $\rho>0$

$$
\int_{C[a, b]} F^{\sharp}\left(\rho^{-\frac{1}{2}} x\right) d x=\rho^{\frac{1}{2}} \int_{0}^{\infty} e^{-\rho s} d f_{1}^{\sharp}(s) .
$$

Hence $\rho^{\frac{1}{2}} \int_{0}^{\infty} e^{-\rho s} d f_{1}^{\sharp}(s)$ has an analytic extension in the half plane $\operatorname{Re} \rho>0$, continuous for $\operatorname{Re} \rho \geqq 0$, and so the limiting $M$ Feynman integral of $F^{*}$ exists for $M=1$ which concludes proof of theorem.

Next we will show that for appropriate functions $\theta$ and $\sigma$, the analytic Wiener and limiting $M$ Feynman integrals of the functional

$$
F(t, \xi, x)=\exp \left[\int_{0}^{t} \theta[t-s, x(s)+\xi] d s\right] \sigma[x(t)+\xi]
$$

satisfy a certain integral equation. 
Theorem 5.2. Assume $\theta(t, \xi)$ is bounded and continuous on $\left[0, t_{\mathrm{v}}\right] \otimes(-\infty, \infty)$ and satisfies for all $t \in\left[0, t_{0}\right]$

$$
\int_{-\infty}^{\infty}|\theta(t, \xi)| d \xi \leqq B
$$

Assume $\sigma(\xi)$ is bounded and continuous on $(-\infty, \infty)$ and satisfies

$$
\int_{-\infty}^{\infty}|\sigma(\xi)| d \xi \leqq B
$$

Then the analytic Wiener integral

$$
G(t, \xi, \lambda)=\int_{C\left[0, t_{0}\right]}^{a n w_{\lambda}} F(t, \xi, x) d x
$$

exists for $(t, \xi, \lambda) \in H=\left(0, t_{0}\right) \otimes(-\infty, \infty) \otimes\{\operatorname{Re} \lambda \geqq 0\}$ and satisfies in $H$ the integral equation

$$
\begin{aligned}
& G(t, \xi, \lambda)=\left(\frac{\lambda}{2 \pi t}\right)^{1 / 2} \int_{-\infty}^{\infty} \sigma(u) \exp \left(-\frac{\lambda(\xi-u)^{2}}{2 t}\right) d u \\
& \quad+\left(\frac{\lambda}{2 \pi}\right)^{1 / 2} \int_{0}^{t}(t-s)^{-1 / 2} d s \int_{-\infty}^{\infty} \theta(s, u) G(s, u, \lambda) \exp \left(-\frac{\lambda(\xi-u)^{2}}{2(t-s)}\right) d s
\end{aligned}
$$

with boundary condition

$$
\lim _{t \rightarrow 0+} G(t, \xi, \lambda)=\sigma(\xi)
$$

holding for all $(\xi, \lambda) \in(-\infty, \infty) \otimes\{\operatorname{Re} \lambda>0\}$.

Proof. The existence of $G(t, \xi, \lambda)$ for all points of $H$ follows from Theorem 8.2 of [2]. Moreover by (0.11) of [2] and the continuity of the functions involved we have

$$
G(t, \xi, \lambda)=\int_{C[0, t]} \exp \left[\int_{0}^{t} \theta\left[t-s, \lambda^{-\frac{1}{2}} x(s)+\xi\right] d s\right] \sigma\left[\lambda^{-\frac{1}{2}} x(t)+\xi\right] d x
$$

for $\lambda>0$ and $(t, \xi) \in H_{1}=\left(0, t_{0}\right) \otimes(-\infty, \infty)$.

Next we note that by use of Theorem 7 on page 66 of [3] (which can be proved for $n=1$ under weakened conditions on $\sigma^{\sharp}$ and $\left.\theta^{\sharp}\right)$ we have that if $\sigma^{\sharp}\left(\xi^{\sharp}\right)$ and $\theta^{\sharp}\left(t, \xi^{\sharp}\right)$ are bounded and continuous for $\left(t, \xi^{\sharp}\right) \in H_{2}=\left[0, t_{0}\right] \otimes(-\infty, \infty)$ then

$$
G^{\sharp}\left(t, \xi^{\sharp}\right)=\int_{C[0, t]} \exp \left[\int_{0}^{t} \theta^{\sharp}\left[t-s, x(s)+\xi^{\sharp}\right] d s\right] \sigma^{\sharp}\left[x(t)+\xi^{\sharp}\right] d x
$$

exists on $H_{2}$ and on $H_{1}$ satisfies 


$$
\begin{aligned}
& G^{\sharp}\left(t, \xi^{\sharp}\right)=(2 \pi t)^{-1 / 2} \int_{-\infty}^{\infty} \sigma^{\sharp}(u) \exp \left(-\frac{\left(\xi^{\sharp}-u\right)^{2}}{2 t}\right) d u \\
& \quad+(2 \pi)^{-1 / 2} \int_{0}^{t}(t-s)^{-1 / 2} d s \int_{-\infty}^{\infty} \theta^{\sharp}(s, u) G^{\sharp}(s, u) \exp \left(-\frac{\left(\xi^{\sharp}-u\right)^{2}}{2(t-s)}\right) d u
\end{aligned}
$$

with boundary condition

$$
\lim _{t \rightarrow 0^{+}} G^{\sharp}\left(t, \xi^{\sharp}\right)=\sigma^{\sharp}\left(\xi^{\sharp}\right) .
$$

Thus, if for $\lambda>0$ we define $\theta^{\sharp}(t, u) \equiv \theta\left(t, \lambda^{-\frac{1}{2}} u\right), \sigma^{\sharp}(u) \equiv \sigma^{\sharp}\left(\lambda^{-\frac{1}{2}} u\right)$, and $\xi^{\sharp} \equiv \lambda^{-\frac{1}{2}} \xi$ then $\theta^{\sharp}$ and $\sigma^{\sharp}$ are bounded and continuous and so $G^{\sharp}\left(t, \xi^{\sharp}\right)$ as defined by (5.12) exists on $H_{2}$ and on $H_{1}$ satisfies (5.13) and (5.14). But for $\lambda>0, \theta^{*}\left[t-s, x(s)+\xi^{\sharp}\right]=\theta\left[t-s, \lambda^{-\frac{1}{2}} x(s)+\xi\right]$, $\sigma^{\sharp}\left[x(s)+\xi^{\sharp}\right]=\sigma\left[\lambda^{-\frac{1}{2}} x(t)+\xi\right]$, and $G(t, \xi, \lambda)=G^{\sharp}\left(t, \lambda^{\frac{1}{2}} \xi\right)$. Hence substituting into (5.13) we see that for $\lambda>0$ and $(t, \xi) \in H_{1}, G(t, \xi, \lambda)$ satisfies (5.9). Then by use of Corollary to Theorem 8.2 of [2] $G(t, \xi, \lambda)$ is bounded in any closed bounded subset of $\operatorname{Re} \lambda \geqq 0$ for all $(t, \xi) \in H_{1}$ and so by use of (5.6) and (5.7) we see that both sides of (5.9) are analytic functions of $\lambda$ for $\operatorname{Re} \lambda>0$, continuous for $\operatorname{Re} \lambda \geqq 0$ and as (5.9) holds for all $\lambda>0$ it must hold throughout $H$.

Boundary condition (5.10) is verified by noting that

$$
\begin{aligned}
\lim _{t \rightarrow 0^{+}} & \left(\frac{\lambda}{2 \pi}\right)^{1 / 2} \int_{0}^{t}(t-s)^{-1 / 2} d s \\
& \times \int_{-\infty}^{\infty} \theta(s, u) G(s, u, \lambda) \exp \left(-\frac{\lambda(\xi-u)^{2}}{2(t-s)}\right) d u=0
\end{aligned}
$$

and

$$
\begin{aligned}
& \left|\left(\frac{\lambda}{2 \pi t}\right)^{1 / 2} \int_{-\infty}^{\infty} \sigma(u) \exp \left(-\frac{\lambda(\xi-u)^{2}}{2 t}\right) d u-\sigma(\xi)\right| \\
& =\left|\left(\frac{\lambda}{2 \pi t}\right)^{1 / 2} \int_{-\infty}^{\infty}[\sigma(u+\xi)-\sigma(\xi)] \exp \left(-\frac{\lambda u^{2}}{2 t}\right) d u\right| \\
& \quad \rightarrow 0 \text { as } t \rightarrow 0^{+} .
\end{aligned}
$$

THEOREM 5.3. Assume $\theta(t, \xi)$ satisfies the hypotheses of Theorem 5.1 where $[a, b]=\left[0, t_{0}\right]$. Assume $\sigma(\xi)$ satisfies the hypotheses of Theorem 5.2. Then for $M=1,2, \cdots$, the limiting $M$ Feynman integral

$$
\hat{G}(t, \xi, q)=\int_{C[0, t]}^{\rightarrow f_{q}^{M}} F(t, \xi, x) d x
$$

where $F(t, \xi, x)$ is defined by (5.5), exists for

$$
(t, \xi, q) \in \hat{H}=\left(0, t_{0}\right) \otimes R_{1} \otimes\left(R_{1}-\{0\}\right)
$$


and satisfies in $\hat{H}$ the integral equation (1.2). Furthermore if we assume $\operatorname{Var}_{(-\infty, \infty)} \sigma(\xi) \leqq B$ then

$$
\lim _{t \rightarrow 0^{+}} \hat{G}(t, \xi, q)=\sigma(\xi) \text {. }
$$

Proof. The existence of (5.16) for $M=1,2, \cdots$, and all $(t, \xi, q) \in \hat{H}$ follows from Theorem 5.1.. But by use of Theorem 2.2 we see that $\widehat{G}(t, \xi, q)=G(t, \xi,-q i)$ where $G$ is defined by (5.8). Then substituting into (5.9) establishes (1.2).

To establish (5.17) first note that

$$
\lim _{t \rightarrow 0^{+}}\left(\frac{-i q}{2 \pi}\right)^{1 / 2} \int_{0}^{t}(t-s)^{-1 / 2} d s \int_{-\infty}^{\infty} \theta(s, u) \widehat{G}(s, u, q) \exp \left(\frac{q i(\xi-u)^{2}}{2(t-s)}\right) d u=0
$$

for all $\xi, q \in R_{1}, q \neq 0$. Also by contour integration and integration by parts one obtains

$$
\begin{aligned}
& \left(\frac{-i q}{2 \pi t}\right)^{1 / 2} \int_{\xi}^{\infty} \sigma(u) \exp \left(\frac{q i(\xi-u)^{2}}{2 t}\right) d u \\
& \quad=\frac{\sigma(\xi)}{2}+\left(\frac{1}{2 \pi}\right)^{1 / 2} \int_{\xi}^{\infty}\left[\int_{-(i q / t)^{1 / 2}(u-\xi)}^{(-i q / t) 1 / 2 \infty} \exp \left(-\frac{z^{2}}{2}\right) d z\right] d \sigma(u) \\
& \quad \rightarrow \frac{\sigma(\xi)}{2} \text { as } t \rightarrow 0^{+} \text {by dominated convergence. }
\end{aligned}
$$

Similarly

$$
\left(\frac{-i q}{2 \pi t}\right)^{1 / 2} \int_{-\infty}^{\xi} \sigma(u) \exp \left(\frac{q i(\xi-u)^{2}}{2 t}\right) d u \rightarrow \frac{\sigma(\xi)}{2} \quad \text { as } t \rightarrow 0^{+} .
$$

REMARK. In view of the remark at the end of $\S 4$ we see that the conclusions of Theorem 5.1 will hold for $M=0$ under the additional assumption that $g^{\prime}$ is of bounded variation on every bounded interval and satisfies

$$
\int_{-V}^{V}\left|d g^{\prime}(w)\right| \leqq B\left[1+|V|^{d}\right]
$$

Also the conclusion of Theorem 5.3 will hold for $M=0$ under the additional assumption that $\sigma^{\prime}$ is of bounded variation on every bounded interval and satisfies

$$
\int_{-V}^{V}\left|d \sigma^{\prime}(w)\right| \leqq B\left[1+|V|^{d}\right]
$$

The author wishes to express his gratitude to Professor Robert Cameron for his encouragement and valuable advise . 


\section{BIBLIOGAPHY}

1. R. H. Cameron, A family of integrals serving to connect the Wiener and Feynman integrals, J. Math. and Physics 39 (1960), 126-140.

2. - The Ilstow and Feynman integrals, J. D'Analyse Mathematique 10 (196263), $287-361$.

3. - Nonlinear Volterra functional equations and linear parabolic differential systems, J. D'Analyse Mathematique 5 (1956), 136-182.

4. R. A. Kallman, On using the Ilstow integral to solve a certain partial differential system, (Submitted for publication)

Received April 28, 1967. 


\section{PACIFIC JOURNAL OF MATHEMATICS}

\section{EDITORS}

\author{
H. ROYDEN \\ Stanford University \\ Stanford, California \\ R. R. Phelps \\ University of Washington \\ Seattle, Washington 98105
}

J. DUGUNDJI

Department of Mathematics

University of Southern California

Los Angeles, California 90007

RICHARD ARENS

University of California

Los Angeles, California 90024

\section{ASSOCIATE EDITORS}
E. F. BECKENBACH
B. H. NeumanN
F. WOLF
K. YosidA

\section{SUPPORTING INSTITUTIONS}

UNIVERSITY OF BRITISH COLUMBIA

CALIFORNIA INSTITUTE OF TECHNOLOGY

UNIVERSITY OF CALIFORNIA

MONTANA STATE UNIVERSITY

UNIVERSITY OF NEVADA

NEW MEXICO STATE UNIVERSITY

OREGON STATE UNIVERSITY

UNIVERSITY OF OREGON

OSAKA UNIVERSITY

UNIVERSITY OF SOUTHERN CALIFORNIA
STANFORD UNIVERSITY

UNIVERSITY OF TOKYO

UNIVERSITY OF UTAH

WASHINGTON STATE UNIVERSITY

UNIVERSITY OF WASHINGTON

AMERICAN MATHEMATICAL SOCIETY CHEVRON RESEARCH CORPORATION TRW SYSTEMS

NAVAL WEAPONS CENTER

Printed in Japan by International Academic Printing Co., Ltd., Tokyo, Japan 


\section{Pacific Journal of Mathematics}

\section{Vol. 26, No. $1 \quad$ November, 1968}

Efraim Pacillas Armendariz, Closure properties in radical theory......... 1

Friedrich-Wilhelm Bauer, Postnikov-decompositions of functors .......... 9

Thomas $\mathrm{Ru}-$ Wen Chow, The equivalence of group invariant positive definite

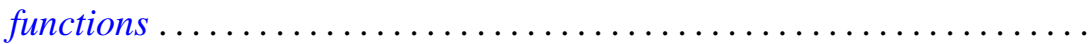

Thomas Allan Cootz, A maximum principle and geometric properties of

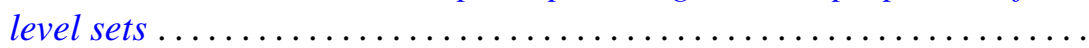

Rodolfo DeSapio, Almost diffeomorphisms of manifolds ............ 47

R. L. Duncan, Some continuity properties of the Schnirelmann density......

Ralph Jasper Faudree, Jr., Automorphism groups of finite subgroups of

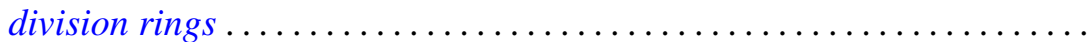

Thomas Alastair Gillespie, An invariant subspace theorem of $J$.

Feldman.........................................

George Isaac Glauberman and John Griggs Thompson, Weakly closed direct factors of Sylow subgroups .............................

Hiroshi Haruki, On inequalities generalizing a Pythagorean functional equation and Jensen's functional equation .....................

David Wilson Henderson, D-dimension. I. A new transfinite dimension.....

David Wilson Henderson, D-dimension. II. Separable spaces and

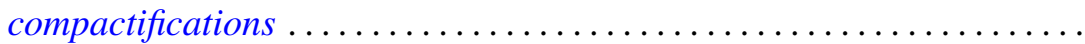

Julien O. Hennefeld, A note on the Arens products ............... 115

Richard Vincent Kadison, Strong continuity of operator functions ...

J. G. Kalbfleisch and Ralph Gordon Stanton, Maximal and minimal coverings of $(k-1)$-tuples by $k$-tuples.

Franklin Lowenthal, On generating subgroups of the Moebius group by pairs of infinitesimal transformations...

Michael Barry Marcus, Gaussian processes with stationary increments possessing discontinuous sample paths . .

Zalman Rubinstein, On a problem of Ilyeff ...

Bernard Russo, Unimodular contractions in Hilbert space. ...

David Lee Skoug, Generalized Ilstow and Feynman integrals...

William Charles Waterhouse, Dual groups of vector spaces . 